\title{
Bionanotechnology: small can have a big impact in the medical sciences: a WIN-win situation. Part I.
}

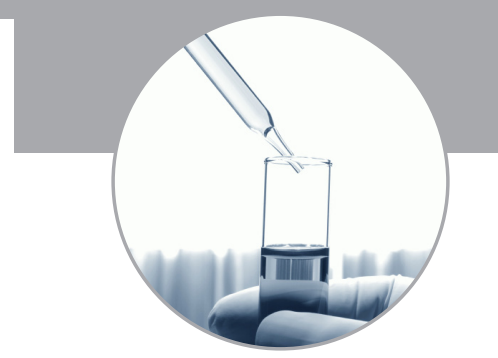

Bionanotechnology blends the areas of nanotechnology and biological sciences. It focuses on a wide area of fundamental research and engineering such as the fabrication of nanomaterials utilizing components and catalysts from the biological sciences as well as the application of nanotechnology to understanding and quantifying biological systems. Areas such as the development of nanomaterial-based drug-delivery systems, the development of novel biosensors and their application to clinical diagnostics as well as high-throughput screening and the exploration of how nanomaterials interact with living systems are all subjects of intense current interest. The Waterloo Institute for Nanotechnology (WIN), coordinates various leading-edge nanotechnology research areas at the University of Waterloo (Canada). One focus is the area of bionanotechnology.

It can be said, as a general rule of thumb, that a new technology has 'arrived' when its potential to drive innovation and economic growth is recognized by the creation and design of national and regional strategies to foster its development. This was true, for example, with information technology and biotechnology. In this sense, nanotechnology has not only emerged but is now expected to be a key driver of industrial innovation in the years ahead. More than 50 national, state and regional governments in developed and developing economies have endorsed 'Nanotechnology Strategies' over the past 10 years. The figures on $R \& D$ investments in nanotechnology bear out this potential. In 2008 , total R\&D investment in nanotechnology reached US $\$ 18.2$ billion, with industrial expenditures of US $\$ 8.6$ billion passing government investments of US $\$ 8.4$ billion for the first time. Venture capital contributed US $\$ 1.2$ billion. The compound annual growth rate for 2007-2008 was $14 \%$. Lux Research projects global marketplace revenues for nano-enabled products by 2015 to be US $\$ 1.5$ trillion, a figure larger than the entire GDP of Canada!

Although Canada lacks, as of yet, a coordinated and synergistic national strategy for nanotechnology, initiatives such as the newly created (2008) Waterloo Institute for Nanotechnology (WIN) will help ensure that Canada can compete at the forefront of the evolving nanotechnology revolution. WIN is an interdisciplinary institute that draws its membership from faculty researchers, post-doctoral fellows and students in nine different departments in science, engineering and mathematics. Its vision is to be 'an interdisciplinary centre of excellence pioneering high-impact research and innovation in nanotechnology'. The full range of assets of WIN includes:

- A total of 55 Faculty in seven theme area clusters of excellence as illustrated in Figure I. Within this group:

- A total of 15 members hold research chair positions (Canada Research Chairs, NSERC Industrial Chairs and Ontario Research Chairs).

- Of 21 new nanotechnology faculty positions created through the Nanotechnology Engineering Undergraduate Program (vide infra), 18 have been filled to date, mostly at the assistant professor level. Thus, WIN has a young and dynamic research community.

- Three Endowed Research Chairs funded by an anonymous donor and matched by the University of Waterloo.

- A US\$10 million endowment for nanofellowships for graduate students, valued at US $\$ 10,000$ to attract and reward outstanding students from around the world to nanotechnology at Waterloo.

- A new quantum nano building (Figure 2), currently under construction, due to be completed in 2011, with 284,000 square feet of space shared equally by WIN and the Institute for Quantum Computing (IQC). The two co-located institutes will share a common suite of state of the art clean rooms, fabrication and metrology facilities to enable a broad cross section of nanotechnology activities and collaborative programs.

John F Honek $\mathbf{k}^{\dagger 1}$,
Alain Francq
\& Arthur J Carty
'Professor \& Chair, Department of
Chemistry, University of Waterloo,
200 University Avenue, Waterloo,
Ontario, Canada, N2L 3GI
2Waterloo Institute for
Nanotechnology, University of
Waterloo, 200 University Avenue,
Waterloo, Ontario, Canada N2L 3GI
†Author for correspondence:
Tel.: + 5198884567
Fax: + 5197460435
E-mail: jhonek@uwaterloo.ca

John F Honek ${ }^{\dagger 1}$,

Alain Francq ${ }^{2}$

\& Arthur J Carty

'Professor \& Chair, Department of Chemistry, University of Waterloo,

${ }^{2}$ Waterloo Institute for

Nanotechnology, University of

tAuthor for correspondence:

Fax: + I 5197460435 

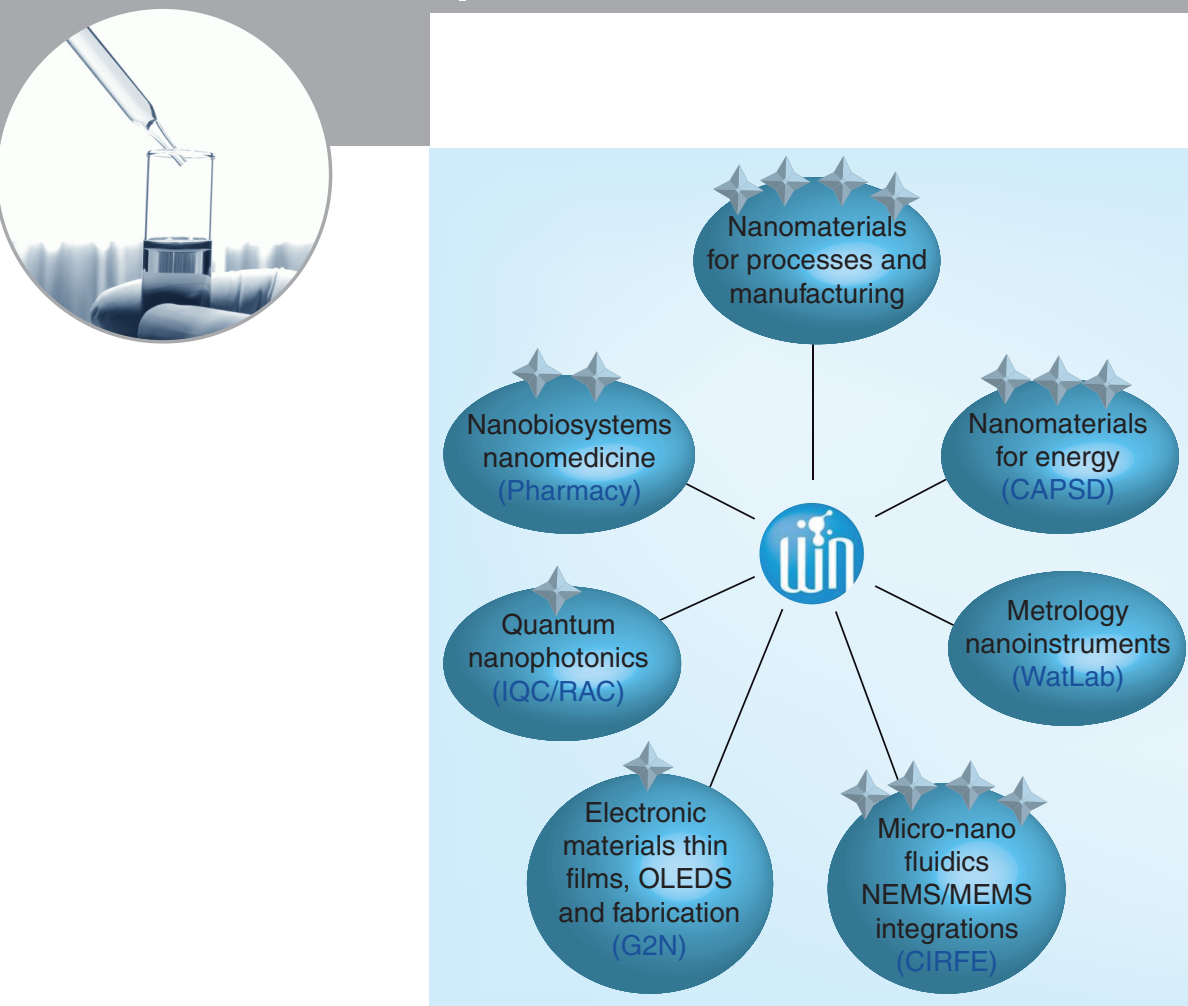

15 research chair holders

(CRC, NSERC, IRC)

- Nanobiomaterials

- Micro- and nano-fluidics

- Nanomedicine and bionanotechnology

- Advanced micro and nano devices

- Micro-/nano-joining

- Spectroscopy of nanoscale materials

- Solid-state nano materials

- Organic electronics

- Solid-state chemistry

- Radio-frequency controlled microelectromechanical system devices

- Advanced rubber technology

- Catalysts and hydrocarbon processing

- Lab-on-a-chip devices

- Renewable energy technologies and health

- Quantum information processing

Figure 1. Research theme areas and research chairs at Waterloo Institute for Nanotechnology (WIN).

CRC: Canada Research Chair; IRC: Industrial Research Chair; NSERC: Natural Sciences and Engineering Research Council.

- In addition, WIN faculty already has access to major $\mathrm{R} \& \mathrm{D}$ infrastructure such as the Giga-to-Nano (G2N) Laboratory, the Centre for Integrated RF Engineering, a major MEMS fabrication lab, the Centre for Advanced Photovoltaic Devices and Systems (CAPDS) and the WATLAB, a characterization facility carrying a broad spectrum of electron and $\mathrm{x}$-ray spectroscopies and microscopy tools for identifying, characterizing and measuring materials on the nanoscale.

- The Quantum Nano Centre building will also house North America's largest coop nanotechnology engineering Program of 500 top students in a 5-year program of academic (3 years) and industrial research (2 years) experience.

Among the many research interests of WIN members (FIGURE 3 ) are activities that fall within the area broadly defined as nanotechnology/nanomedicine. This refers to the application of nanotechnology to achieve breakthroughs in healthcare and diagnosis, targeted treatment, therapy, tissue repair and regeneration. Nanomedicine exploits the novel physical, chemical and biological properties of materials on the nanoscale $\left(10^{-9} \mathrm{~m}\right)$ to address urgent clinical needs. TABLE I lists WIN researchers in this nanobio area and provides keywords describing their interests.

\section{Bionanotechnology focus at WIN}

Although a substantial amount of biological and medicinal chemistry is undertaken at the University of Waterloo, the bionanotechnology members associated with the WIN contribute to investigations on the application of nanotechnology to medical diagnostics and therapeutics. The group is composed of Marc Aucoin (chemical engineering), Melanie Campbell (physics), $\mathrm{Pu}$ Chen (chemical engineering), Marianna Foldvari (pharmacy), Frank Gu (chemical engineering), John Honek (chemistry), Zoya Leonenko (biology/physics), Juewen Liu (chemistry), Vivek Maheshwari (chemistry), Michael Tam (chemical engineering) and Shirley Tang (chemistry), and their research laboratories. A brief survey of some of the member's research is presented below along with the technologies that they are exploring.

\section{Drug carrier \\ \& drug-delivery technologies}

Development of novel nanodelivery systems is one prominent focus of the bionanotechnology WIN group. The successful development of novel drug-delivery technologies will provide improved drug and gene therapies through better targeting and localization of drugs at disease sites, offer new options to utilize drug molecules that could not be delivered before and provide alternative routes for delivery. 

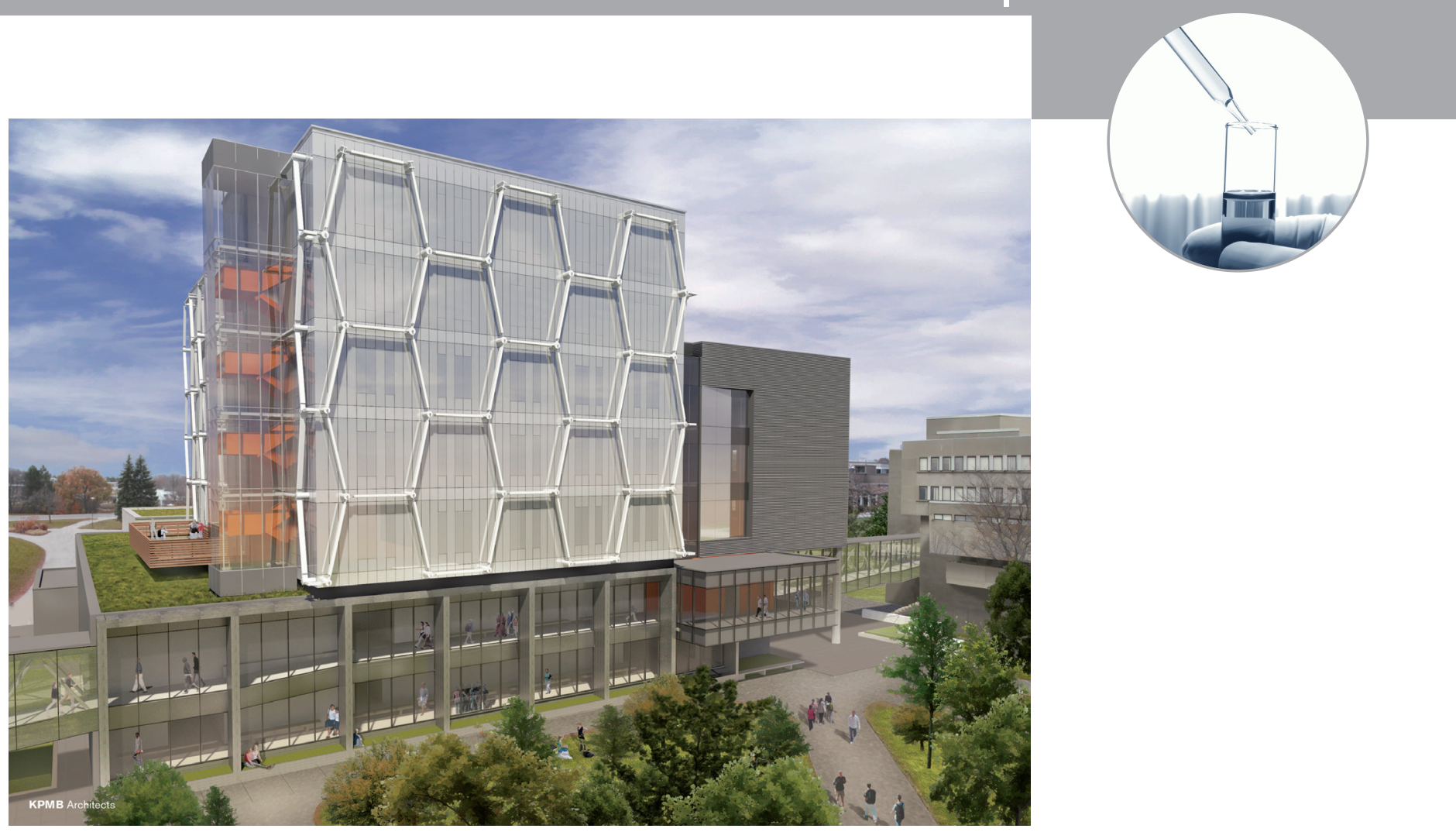

Figure 2. The Quantum Nano Centre.

Reproduced with permission from KPMB Architects.

Work in the Chen laboratory is concerned with peptide-mediated drug and gene delivery and focuses on two classes of peptides: selfassembling peptides (SAPs) and cell-targeting peptides (CTPs), in relation to the development of nanocarrier delivery systems [1-3]. Selfassembling peptides are emerging in a wide range of biomedical and bioengineering applications and fall into several classes, including peptide amphiphilies, bolaamphiphile peptides, cyclic peptides and ionic complementary peptides. These peptides can be found naturally or can be synthesized. The advantage of synthesized peptides is that the self-assembling properties can be exploited to form desirable structures for various functions. The design principles of well-known self-assembling peptides, as well as their physical/chemical properties, are part of Chen's research effort. A unique property of self-assembling peptides is their stimuli-responsiveness in different environments including $\mathrm{pHs}$, temperatures and ionic strengths. This characteristic makes peptides applicable to a wide range of biomaterials for drug discovery. In addition, therapeutic cancer-targeting peptides and current combinatorial peptide library methods, which are used to identify targeting peptides, are being pursued. Cancer-targeting
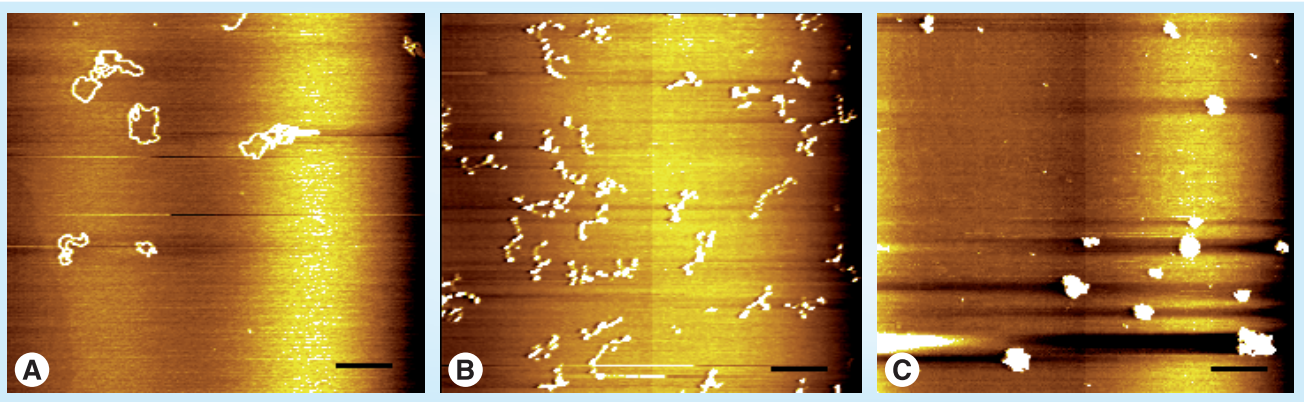

Figure 3. Atomic-force microscopic images of the compaction of plasmid DNA into nanoparticles by gemini surfactant 16-3-16. (A) 'Naked' plasmid. (B) Initial phases of compaction $30 \mathrm{~s}$ after adding gemini surfactant; bar $100 \mathrm{~nm}$. (C) Nanoparticle formation $15 \mathrm{~min}$ after adding gemini surfactant; bars $400 \mathrm{~nm}$.

Reproduced with permission from [12]. 
Table 1. Researchers and research interests in nanobiotechnology at WIN.

\section{Specific research programs}

Virus-based systems as novel nanobiomaterials; complex biologics

Optical properties of the eye; biophotonics; studies of amyloid- $\beta$ at the nanoscale

Targeted drug and gene delivery; interfacial engineering and polymer synthesis

Novel nanomaterials and strategies for transdermal drug delivery

Nanoparticles in medicine; targeted drug delivery

Application of biological chemistry to synthesis of new nanomaterials and structures

Biophysics of lipids; application of lipid films in biomedical nanotechnology

Targeted drug delivery, nanomedicine and self-assembly

Nanoparticle-based thin-film sensors; materials and tools for bionanodevices

Nanosystems for drug delivery, polymer-surfactant interactions and

magnetic nanoparticles

Nanomaterials; devices for biology and medicine

Surfaces and interactions of soft materials at the nanoscale

${ }^{+}$Canada Research Chair.

peptides can target either tumor cell surfaces or tumor vasculatures. The RGD peptide is one of the first tumor-targeting peptides found that can bind to self-assembling peptides or any other nanocarrier to improve the therapeutic efficiency of drug-delivery systems.

Foldvari's research focus is threefold in nature. One research initiative is the development of methodologies for gene therapy using nonviral gene-delivery vehicles. Gene therapy is a medical technique for the correction of genetic defects by inserting a normal gene as a replacement for a faulty gene or correcting (e.g., turning off) the function of an overexpressing gene. Presently, the delivery efficiency of viral vectors is higher than nonviral systems and a significant number of clinical trials involve adenovirusbased systems. Unfortunately, immunogenicity, inflammatory reactions, the maximum length of gene, certain concerns about viral vectors causing disease and complicated manufacturing and purification places many limitations on their clinical application. Therefore, the development of nonviral delivery systems with hightransfection efficiency is imperative for realizing the potential benefits of gene therapy.

Foldvari's group has developed gemininanoparticles based on a novel class of dicationic gemini surfactants as nonviral nucleic acid delivery systems $[4,5]$. When the DNA plasmid is combined with gemini surfactants (PG) and another 'helper' lipid, dioleoylphosphatidylethanolamine or DOPE (PGL), nanoparticles with an average size of 100-200 $\mathrm{nm}$ form (Figure 3). Gemini nanoparticles have the advantage of generally having low toxicity/immunogenicity, as well as no limitations with regard to the size of DNA that can be delivered. Characterization of the structural and physicochemical properties of these dicationic lipid-based DNA complexes by small-angle $\mathrm{x}$-ray scattering (SAXS), zeta potential and particle size analysis indicate correlation between polymorphic flexibility of the nanoparticles and cellular transfection efficiency.

During this self-assembly process, several important physicochemical changes take place with regards to the plasmid DNA:

- The DNA is compacted into smaller particles compared to its original state;

- The surface charge of the DNA changes from negative to positive (+ zeta potential);

- The internal DNA-gemini surfactant structural arrangement takes on a mixed polymorphic form.

All these properties were found to be important in achieving successful transfection both in vitro and in vivo.

Carbon nanotubes (CNTs) have many potential applications as pharmaceutical excipients. Their unusual properties, in particular their extreme length-to-diameter ratio, propensity for chemical functionalization and potential biocompatibility, make CNTs promising candidates as delivery vehicles for biologically active molecules, as targets for biophysical treatments and as templates for tissue growth and regeneration (Figure 4). As CNTs are hollow, they also have the potential to transport drugs by nanofluidic delivery $[6,7]$. Here we show the time course of delivery of ansiRNA-multiwall carbon nanotube (MWNT)-complex into PAM212 keratinocytes by confocal microscopy (FIGURE 5). 
(A)

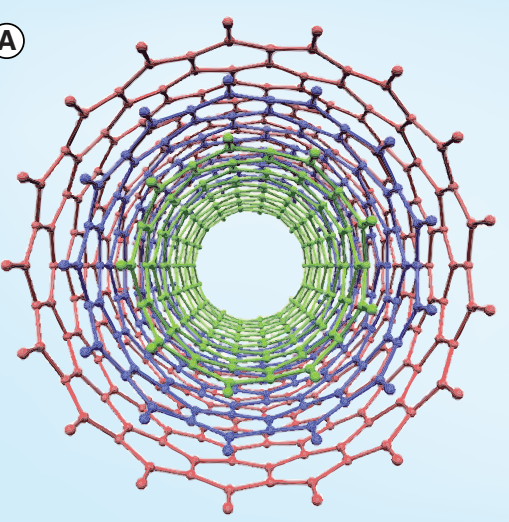

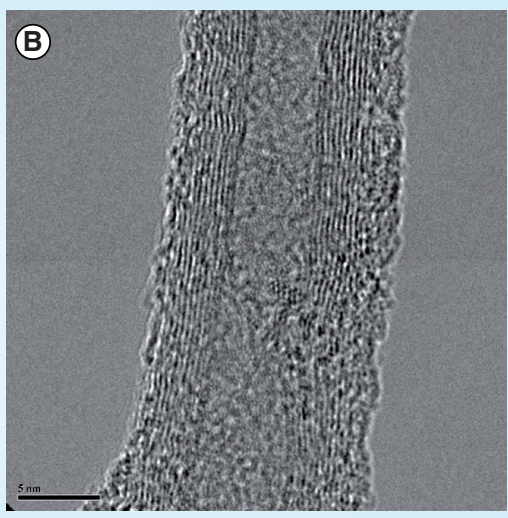

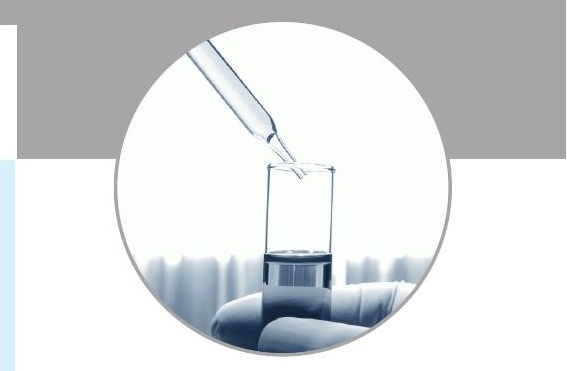

Figure 4. Schematic (A) and transmission electron microscopic image (B) of a multiwall carbon nanotube ( $15 \mathrm{~nm}$ outer diameter) synthesized using the SSP-354 benchtop chemical vapor deposition system by Nanotech Innovations.

The Foldvari group has also developed 'biphasic vesicles' (Biphasix ${ }^{\mathrm{TM}}$ ), which represent a platform drug-encapsulation technology suitable for delivering small-drug molecules, proteins and vaccines [8]. The Biphasix technology is a novel microscopic delivery system that could replace needles in the administration of proteins and genes, the pharmaceutical drugs of the future. Such large molecules can generally only be administered by painful injections and may not attain full therapeutic potential because they degrade before reaching the disease site. The first application of the Biphasix is a topical interferon preparation currently in Phase II clinical trials for the treatment of cervical and ano-genital human papilloma virus (HPV) infections. A treatment currently approved for HPV infections is intramuscular and intralesional interferon- $\alpha 2 b$ (IFNo, Intron A), a 19-kDa protein with antiviral, antiproliferative and immunomodulatory properties. The goal was to develop biphasic vesicles (Biphasix) as a noninvasive topical delivery system for IFNa. In one of several Phase II studies, 41 women with cytologically confirmed,
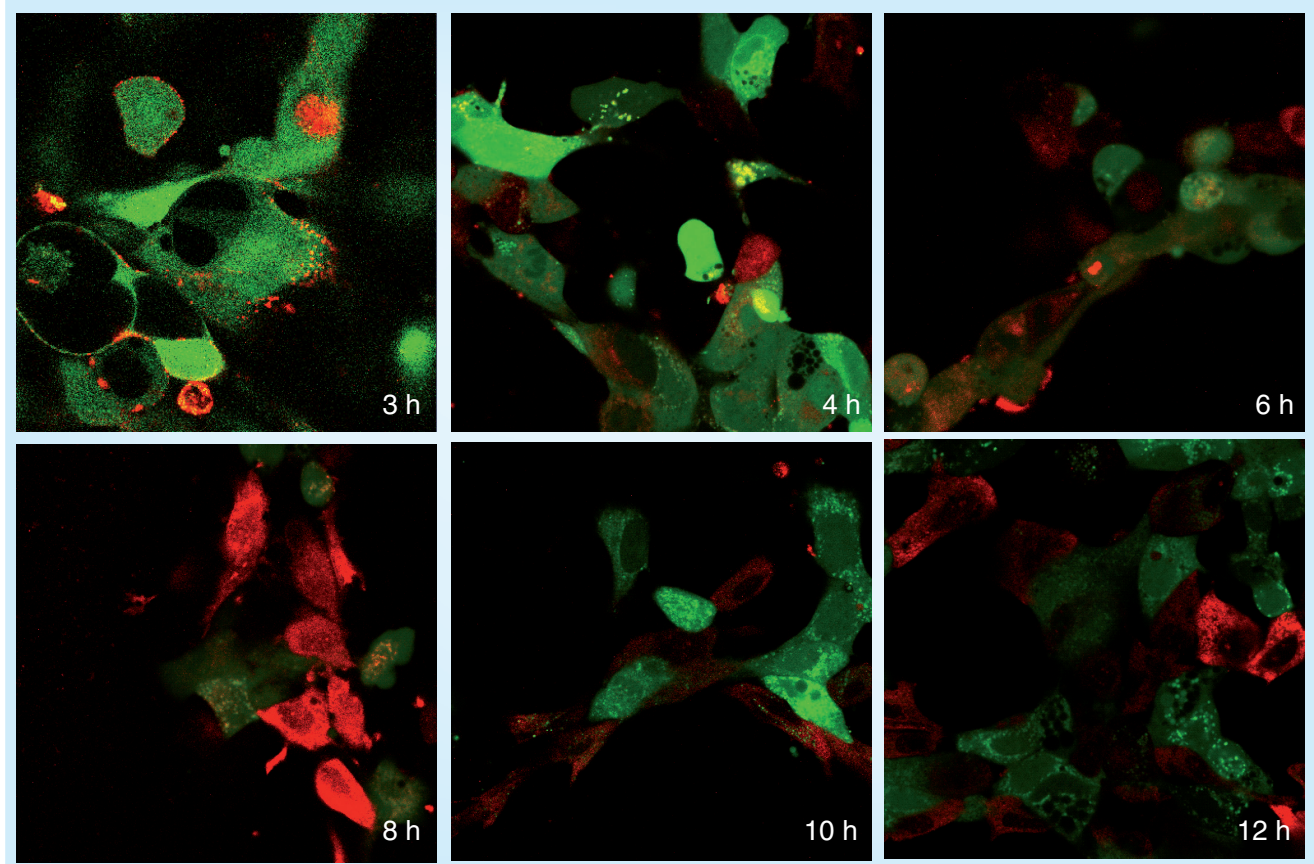

Figure 5. Time-course of intracellular delivery of siRNA by multiwall carbon nanotubes into murine PAM 212 keratinocytes. Cell viability is indicated by calcein staining (green); siGLO-RNA uptake into cells is indicated by red. 


\section{News \& Analysis | Research SpOTlight}
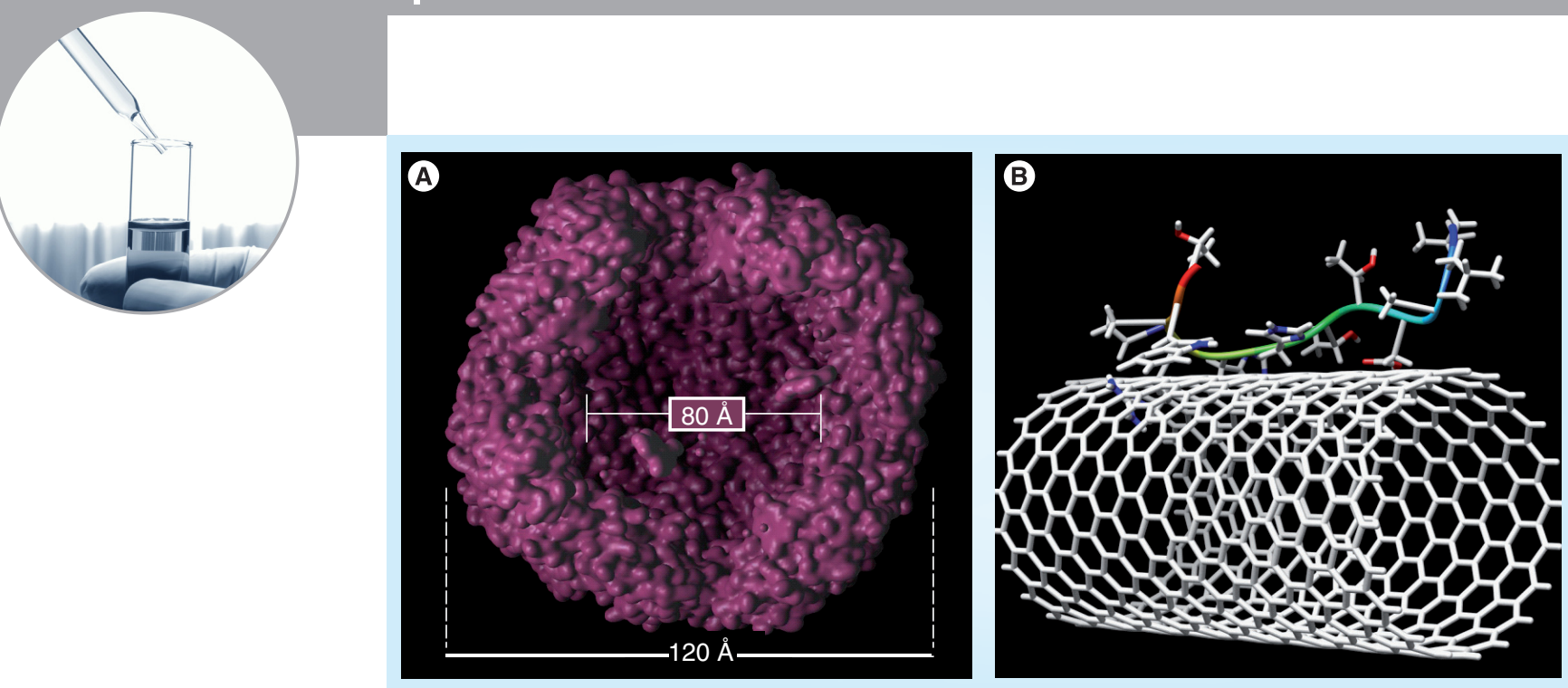

Figure 6. Bacterioferritin and Peptide-Nanotube Interactions. (A) Visualized internal cavity of bacterioferritin showing the nanoscale dimension of this class of protein. (B) Peptide-single-walled nanotube interaction based on various biophysical studies conducted by the Honek group.

HPV-induced low-grade squamous intraepithelial lesions (LSIL, PapIIw to Pap IIID) of the cervix were studied across four clinical sites. In the treatment group 20 women received topical Biphasix-IFNa-2MU, self-administered intravaginally three times per week for a period of 6 weeks with a follow-up evaluation at 12 weeks. In the placebo group, 21 women received no treatment. The preclinical development of BiphasixIFN $\alpha$ indicated excellent dermal safety and product stability. Clinical trial results in patients with LSIL/CIN1 indicated that $46.7 \%$ of the women in the treated per-protocol population had their abnormal Pap smears revert to normal during the 12 -week period, compared with only $15.8 \%$ of the untreated women. A second Phase II pharmacokinetic study in ten patients with LSIL/CIN1 have indicated low systemic exposure (below the lower limit of detection $6.25 \mathrm{pg} / \mathrm{ml}$ ) after topical treatment. Another double-blind, placebo-controlled multicenter Phase II trial in 129 female patients with external ano-genital warts treated twice-daily, for five consecutive days per week for 8 weeks is currently ongoing.

The Honek group focuses on two areas of bionanotechnology. The first is the investigation of the mechanism and control of the self-assembly of multisubunit proteins and their use in encapsulation techniques. The group is interested in investigating the controlled encapsulation of guest atoms/molecules into the nanometer-sized cavities $(5-10 \mathrm{~nm}$ in diameter) of large $(>400 \mathrm{kDa})$ proteins such as bacterioferritins (Figure 6A). This approach may then permit molecular protection of metabolically sensitive drug molecules and polymers to be delivered to key cell types using
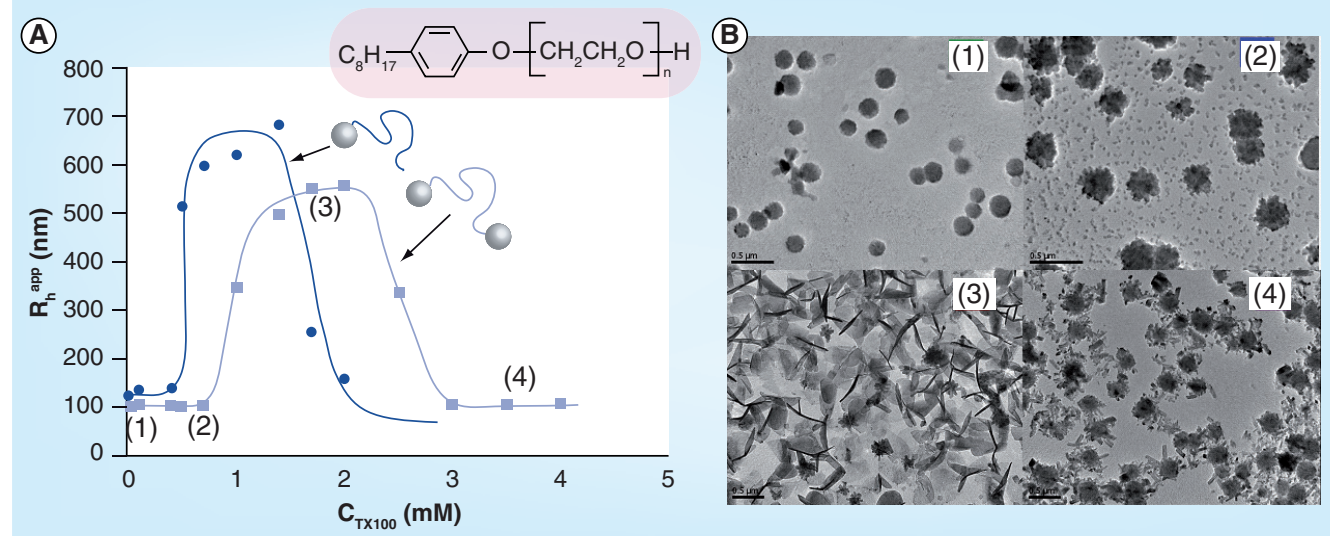

Figure 7. Particle size distributions and micrographs demonstrating the morphology change of unlonized di-end-capped C60-b-PAA83-b-C60 induced by the binding of TX100: (A) $\mathrm{C}_{\mathrm{T} \times 100}=0 \mathrm{mM}$; (B) $\mathrm{C}_{\mathrm{T} \times 100}=0.7 \mathrm{mM}$; (C) $\mathrm{C}_{\mathrm{T} \times 100}=1.7 \mathrm{mM}$; (D) $\mathrm{C}_{\mathrm{T} \times 100}=3.5 \mathrm{mM}$. 
surface-expressed directing groups. These types of complexes may also be utilized in arranging internal guest atoms/molecules into arrays, which might provide new molecular-building blocks for applications in the biosensor area as well. As one can control some of the chemical structures of these biomaterials by recombinant DNA techniques as well as by chemical modification, this approach has great potential for the controlled design of complex nanoscale biomaterials.

A second area of interest is the study of the interaction that biomolecules, such as peptides and proteins have with nanomaterials, metal surfaces and carbon-based nanotubes [9-11]. For example, the group is selecting for peptides that have high affinity for single-walled carbon nanotubes by phagedisplay methods and have studied some of these interactions at the molecular level (Figure 6B). The group uses phage display methods to identify high-affinity binding peptides to nanomaterials. The resulting peptides could be used to control material coatings useful in controlled drug release as well as in optimizing biomolecule-xenomaterial interactions in the human body and may lead to new composite materials important in medicine. The addition of unnatural amino acids into these peptides has been shown to further modify the binding affinities of these peptides.

Other encapsulation technologies are being developed in the research laboratories of Michael Tam. Due to the theoretical importance and potential applications of fullerene, numerous fullerene derivatives have been developed to enhance its solubility and processability. Tam's research at Waterloo focuses on the design and synthesis of fullerene-containing polymers, where interesting nanostructures can be produced and used to encapsulate pharmaceutical compounds. They are also excellent candidates for use as free-radical scavengers and UV blockers in cosmetic formulations. Owing to the unique chemical structure of fullerene, different fullerene-containing polymeric architectures can be synthesized using a variety of conjugating techniques. The Tam group has adopted the azido coupling and atom transfer radical addition strategy to synthesize 'controlled' and well-defined fullerene-containing polymers. Experimental results have indicated that fullerenecontaining polymers not only increase the solubility of fullerene in solution, but also retain the conjugating properties of fullerene molecules. By blocking well-defined functional polymers onto fullerene molecules, different types of stimuliresponsive amphiphilic systems can be achieved. However, the large bulk volume and high hydrophobicity of fullerene gives rise to large aggregates with different morphologies produced in solution, which can be tuned by changing external stimuli, such as $\mathrm{pH}$, temperature, salt and co-solvents. Interestingly, fullerene-containing anionic polymers could induce the formation of nanoscale fractal patterns. Complexation of fullerene polymers with other amphiphilic molecules produces interesting nanostructures that depend on the architecture and the chemical composition of the polymers (FIGURE 7).

\section{Future perspective}

A better understanding of the synthesis and control of nanomaterials will occur in the next 5-10 years, alongside improved techniques to control the size, shape and biocompatibility of biomaterials created by bionanotechnology. Additional information will be provided on their important pharmacokinetic and pharmacodynamic properties and how best to deploy their properties in medical sciences.

\section{Acknowledgments}

Grateful acknowledgment is made to the research text and figures contributed for this article by Professors Chen, Foldvari, Honek and Tam.

\section{Financial \& competing interests disclosure}

The authors would like to thank NSERC (Canada) and the University of Waterloo for financial support. The authors have no other relevant affiliations or financial involvement with any organization or entity with a financial interest in or financial conflict with the subject matter or materials discussed in the manuscript apart from those disclosed.

No writing assistance was utilized in the production of this manuscript.

\section{Key terms}

Phage display: Application of bacteriophage (viral) libraries composed of large numbers of viruses, each displaying a surface peptide/protein of unique sequence. In essence the library is a viral-based peptide library.

Fullerene: Entirely carbonbased molecule, whose structure can form either a sphere, as in $\mathrm{C60}$, an ellipsoid or a tube (such as a single-walled carbon nanotube).

\section{Executive summary}

- Drug encapsulation protocols can vary and can include polymer nanoshells, as well as nanocavities in proteins.

- Surfactant-based drug and gene delivery can be optimized to introduce new clinically useful molecular delivery systems.

Application of phage-display may be used to study the fundamental interactions between nanomaterials and biomolecules but also serve as unique linkers for the attachment of drugs and/or biosensors to material surfaces and prostheses.

- New approaches to fabricating and characterizing nanomaterials allows for improved design of molecular scaffolds, which are tailor-made to the desired application. 


\section{News \& ANalysis | Research Spotlight}

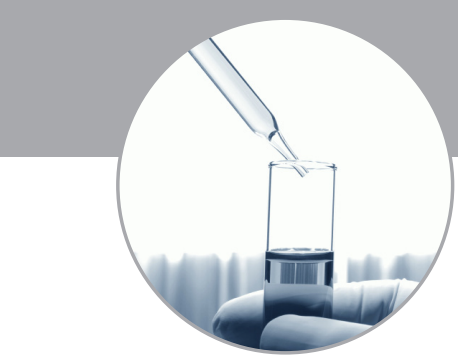

\section{Bibliography}

1 Jafari M, Chen P. Peptide mediated siRNA delivery. Cur. Top. Med. Chem. 9, 1088-1097 (2009).

2 Fung S, Yang H, Bhola PT et al. Self-assembling peptide as a potential carrier for hydrophobic anticancer drug Ellipticine. Adv. Funct. Mater. 18, 1-10 (2008).

3 YangH, Fung S, Pritzker M, Chen P. Mechanical force-induced peptide assembly at liquid/solid interfaces. Angew. Chem. Int. Ed. 47, 4397-4400 (2008).

4 Wettig SD, Verrall RE, Foldvari M. Gemini surfactants: a new family of building blocks for nonviral gene delivery systems. Cur. Gene Ther. 8, 9-23 (2008).

5 Badea I, Wettig S, Verrall R, Foldvari M. Topical noninvasive gene delivery using gemininanoparticles in interferon- $\gamma$-deficient mice. Eur. J. Pharm. Biopharm. 65, 414-422 (2007).

6 Foldvari M. Formulating nanomedicines: focus on carbon nanotubes as novel nanoexcipients. Key Eng. Mater. 441, 53-74 (2010).
7 Foldvari M, Bagonluri M. Carbon nanotubes as functional excipients for nanomedicines: I.

Pharmaceutical properties. Nanomedicine 4, 173-182 (2008).

8 Foldvari M, Badea I, Wettig S et al. Topical delivery of interferon $\alpha$ by biphasic vesicles: evidence for a novel nanopathway across the stratum corneum. Mol. Pharm. 7, 751-762 (2010).

9 Su Z, Mui K, Daub E, Leung T, Honek JF. Interactions of peptides with single-walled carbon nanotubes. Adv. Exp. Med. Biol. 611, 235-236 (2009).

10 Su Z, Mui K, Daub E, Leung T, Honek JF. Single-walled carbon nanotube binding peptides: probing tryptophan's importance by unnatural amino acid substitution. J. Phys. Chem. B 111, 14411-14417 (2007).

11 Su Z, Leung T, Honek JF. Conformational selectivity of peptides for single-walled carbon nanotubes. J. Phys. Chem. B 110, 23623-23627 (2006).

12 Foldvari M, Badea I, Wettig S, Verrall R, Bagonluri M. Structural characterization of novel gemini non-viral DNA delivery systems for cutaneous gene therapy. J. Exp. NanoSci. 1(2), 165-176 (2006). 